\section{Hanseníase em menores de quinze anos no município de \\ Paracatu, MG (1994 a 2001)\#}

\section{Leprosy in patients under fifteen years of age in the city of Paracatu- MG (1994 to 2001)}

\section{Isaias Nery Ferreira ${ }^{1 *}$}

\section{Rosicler Rocha Aiza Alvarez ${ }^{2}$}

${ }^{1}$ Fundação Nacional de Saúde - Funasa, Unidade Mista de Paracatu - MG ${ }^{2}$ Hospital Universitário - Ambulatório de Dermatologia, Universidade de Brasília

"Esta pesquisa foi financiada pelo autor, Isaias Nery Ferreira.

*Correspondência: Rua Afrânio Salustiano Pereira, 303, Bairro Bela Vista, 38600-000

Paracatu - MG, isaias@unb.br

\section{Resumo}

O Município de Paracatu-MG é considerado prioritário para ações de controle da hanseníase devido às suas taxas de detecção e prevalência desta nosologia, sobretudo em menores de quinze anos. Nos anos de $1998 \mathrm{e}$ 1999, realizou-se um trabalho de esclarecimento sobre sinais e sintomas da doença para uma população de cerca de oito mil escolares, o que contribuiu para o incremento da taxa de detecção da doença em menores de quinze anos, sendo o município classificado como hiperendêmico entre os anos de $1997 \mathrm{e}$ 2001. Realizou-se também um trabalho de desenho descritivo de quarenta e cinco pacientes diagnosticados e tratados no município de Paracatu entre 1994 e 2001. Cinqüenta e três por cento dos diagnósticos foram em crianças do sexo masculino, $75,5 \%$ dos casos se situavam entre dez e catorze anos de idade e a escolaridade da população deste estudo está defasada em relação à idade, sendo que $84 \%$ da clientela morava em zona urbana. Todos os inscritos (100\%) foram casos novos, $56,0 \%$ de forma multibacilar, forma clínica Dimorfa, e $44 \%$ de formas paucibacilares, como a forma clinica Indeterminada $(30,8 \%)$ e a Tuberculóide (13,2\%). Todos foram tratados segundo esquema padrão, com $100 \%$ de cura. Vinte e quatro por cento dos doentes apresentaram reações no diagnóstico e durante o tratamento, e 9\% apresentaram reações no pós-alta. Vinte e dois por cento dos doentes apresentavam algum grau de incapacidade no diagnóstico e 13\% apresentavam algum grau de incapacidade na alta (índice considerado alto). Verificou-se que $62 \%$ dos pacientes tinham contatos intradomiciliares portadores de hanseníase, sendo que somente em $15,5 \%$ destes contatos foram verificadas as duas cicatrizes da vacina BCG. A hanseníase é um sério problema de saúde pública no município estudado, e o trabalho realizado com escolares foi fundamental para o aumento da detecção dos casos.

Palavras-chave: Hanseníase. Menores de quinze anos. Paracatu-MG. 


\section{Abstract}

The city of Paracatu-MG is considered a priority for leprosy control actions due to the high rates of detection and prevalence of the condition, especially among children under fifteen years of age. In 1998 and 1999, an information program was implemented in order to provide explanations on the signs and symptoms of the disease to a population of 8,000 students, in order to improve the detection rate of the disease in children under fifteen years of age, given Paracatu was classified as a hyper-endemic city between 1997 and 2001. A descriptive study was carried out comprising forty-five patients diagnosed and treated in Paracatu between 1994 and 2001. Fifty-three percent of diagnoses were in male children, $75.5 \%$ of the cases being between ten and fourteen years of age; individuals had less years of schooling than what would be expected for their ages; $84 \%$ of the population lived in the urban area. All patients included (100\%) were new cases, of which $56 \%$ were multibacillary forms, mainly the Dimorph clinical form (potentially infectious), followed by the Undetermined (30.8\%) and Tuberculoid (13.2\%) forms. All were treated according to standard procedures, with a $100 \%$ cure rate. During treatment $24 \%$ of patients presented reactions, and $9 \%$ did after being discharged. Twentytwo percent of patients were diagnosed with some degree of disability, and $13 \%$ presented some degree of disability upon discharge (a rate considered high). Sixty-two percent of patients had contact with other Hansen's disease bearers, and only $15.5 \%$ of these contacts had two BCG vaccine scars. Leprosy is a serious public health problem in the city studied, and the work accomplished with students was primary to increase case detection.

Key Words: Leprosy. Under fifteen. Paracatu-MG.

\section{Introdução}

O agente causador da hanseníase é o Mycobacterium leprae, um parasita intracelular obrigatório, que ocasiona uma doença de evolução lenta e que se manifesta, essencialmente, através de sinais e sintomas dermatoneurológicos como as lesões de pele e de nervos periféricos, acometendo principalmente olhos, mãos e pés ${ }^{1}$.

A hanseníase ainda representa um sério problema de saúde pública. Com relação ao Brasil, a doença se manifesta de forma preocupante, pois entre os anos de 1985 a 1996, o coeficiente de prevalência da hanseníase diminuiu, enquanto o coeficiente de detecção aumentou ${ }^{2}$. Na $3^{\text {a Conferên- }}$ cia Regional OPAS/OMS sobre a eliminação da hanseníase das Américas, realizada em 1999 na Venezuela, o Brasil assinou a Declaração de Caracas, comprometendo-se novamente a eliminar a hanseníase como problema de saúde pública, desta vez até o ano de 2005, pois não conseguimos atingir a meta da eliminação da doença em nosso país no ano de $2000^{3,4}$

A Figura 1 mostra o comparativo do Coeficiente de Detecção Geral de hanseníase, no Brasil, no estado de Minas Gerais e em Paracatu - MG, entre 1994 e 2001. Observamos nesta figura que a taxa de detecção de hanseníase, no período observado, mantémse constante no Brasil, no estado de Minas Gerais, e em crescimento no município de Paracatu, caracterizando-o como município hiperendêmico ( $>4 / 10.000$ hab.), segundo os indicadores epidemiológicos vigentes. Notase uma elevação substancial deste indicador, no ano de 1999, provavelmente em decorrência de um treinamento em ações de controle da hanseníase, organizado pela equipe local com treinamento dos profissionais de saúde de diversos serviços, principalmente para algumas equipes do Programa de Saúde da Família. Outra atividade que se destacou foi a divulgação de sinais e sintomas da doença para a população, especialmente escolares com a prioridade da detecção precoce desta nosologia.

Este trabalho teve como objetivo estu- 


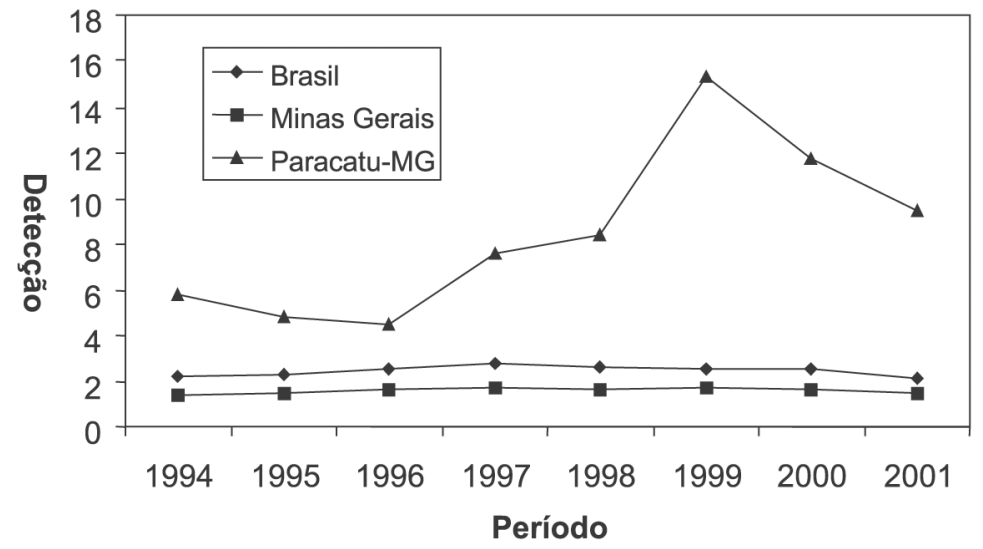

Figura 1 - Comparativo do Coeficiente de Detecção de Hanseníase no Brasil, em Minas Gerais e em Paracatu, entre 1994 e 2001.

Figure 1 - Comparative Detection Coefficient of Leprosy in Brazil, Minas Gerais and Paracatu-MG from 1994 t0 2001.

Fonte: Ministério da Saúde, Secretaria Estadual da Saúde e Centro de Saúde Central, 2002.

Source: Ministry of Health, Minas Gerais State Health Department and Central Health Center. 2002.

dar as características clínicas e epidemiológicas dos pacientes menores de quinze anos, inscritos no serviço de hanseníase, em Paracatu-MG, no período de 1994 a 2001.

\section{A hanseníase em menores de quinze anos}

O problema da hanseníase em menores de quinze anos e suas conseqüências vêm sendo objeto de estudo por parte de diversos autores e organismos internacionais ${ }^{5-8}$.

Apesar da hanseníase ser considerada como doença do adulto e do adulto-jovem, existem numerosos relatos de casos desta enfermidade em faixas etárias menores de quinze anos. A justificativa para este fato é a existência de um aumento na cadeia de transmissão do bacilo na comunidade, além de uma deficiência na vigilância e no controle da doença ${ }^{9,10}$. Em países endêmicos, a população infantil entra precocemente em contato com doentes bacilíferos, sendo possível observar uma detecção da doença entre crianças de três a cinco anos e raramente são observados casos em menores de dois anos, principalmente da forma Virchowiana ${ }^{11-13}$.

As crianças correm maior risco quando existe a presença da hanseníase na família ou quando um caso bacilífero está próximo a elas ${ }^{7,14-16}$.

Os sinais clínicos da hanseníase muitas vezes não são fáceis de serem diagnosticados na infância ${ }^{12}$. A própria idade desta clientela é um fator limitador e, em algumas regiões endêmicas, é elevado o número de crianças com deformidades provocadas pela hanseníase ${ }^{17}$. A importância desses agravos e seus problemas sociais, físicos e desenvolvimento psicológico não podem ser negligenciados devido à possibilidade do próprio futuro dessa clientela estar comprometido ${ }^{18}$.

Quanto ao tratamento, a introdução do esquema padronizado produziu significativa redução na prevalência da doença, especialmente em crianças ${ }^{15}$. Um controle rigoroso, envolvendo a vigilância epidemiológica, deve ser mantido em crianças sob risco de contrair a hanseníase, no sentido de detectar a doença mais precocemente e evitar as conseqüências do diagnóstico tardio ${ }^{17}$ e dos estigmas sociais ${ }^{19}$.

A literatura aponta que a presença da hanseníase em menores de quinze anos é utilizada habitualmente como um indicador do nível de transmissão da doença hansênica. Também existe uma relação entre a proporção de casos em menores de quinze anos e a gravidade da endemia ${ }^{9}$. Ou seja, quando a transmissão é intensa, aumenta a probabilidade do surgimento da doença na população mais jovem, devido à exposição ao bacilo de Hansen nos primeiros anos de vida ${ }^{11}$. 
A taxa de prevalência de hanseníase em crianças pode variar de 3,0 a 45,0\%, de acordo com o nível da endemia de cada região $0^{20,21}$. A detecção de casos novos é elevada nas regiões endêmicas, mas é variável de acordo com o local estudado ${ }^{7,22,23}$.

No Brasil, em 1985, o coeficiente de detecção de hanseníase em menores de quinze anos* era de 0,37/ 10.000 hab., coeficiente considerado alto de acordo com o parâmetro do Ministério da Saúde. Onze anos depois, em 1996, este coeficiente atingiu 0,68/ 10.000 hab., o que foi classificado como muito alto. $\mathrm{O}$ incremento no coeficiente de detecção de casos de hanseníase nesta faixa etária, no período, foi de $84 \%^{2}$. Em 2003, a região Norte apresentou uma detecção de 1,70 (hiperendêmica); as regiões Centro-Oeste e Nordeste apresentaram para esta detecção 0,81 e 0,80, respectivamente, coeficientes considerados muito altos; a região Sudeste apresentou 0,29, o que foi considerado alto, e a região Sul mostrou 0,10 , coeficiente considerado médio ${ }^{4}$.

O município de Paracatu-MG possuía, em 2000, uma população de 75.215 habitantes, sendo $34,6 \%$ menores de quinze anos, e foi considerado prioritário pela Secretaria Estadual de Saúde em ações de controle de hanseníase, devido à condição de ser hiperendêmico, conforme demonstra a taxa de detecção de casos e prevalência da doença em menores de quinze anos. Frente a este cenário, entendeu-se ser necessário analisar a endemia hansênica em Paracatu-MG, seu perfil e, principalmente, suas conseqüências nesta população.

\section{Pacientes e Métodos}

Realizou-se um estudo epidemiológico de natureza descritiva. A população deste estudo foi constituída por quarenta e cinco pacientes portadores de hanseníase, menores de quinze anos, diagnosticados, registrados e tratados no município de Paracatu-MG, no período de 1994 a 2001. Todos os pacientes inscritos no Programa de Hanseníase, no período considerado, terminaram o tratamento, não havendo transferências, abandonos ou óbitos. Não houve perda de dados.

Os pacientes foram diagnosticados obedecendo à classificação de Madri: Forma Indeterminada (I), Tuberculóide (T), Virchowiana (V) e Dimorfa (D). A classificação operacional para fins de tratamento foi de Formas Paucibacilares - PB - (Indeterminada e Tuberculóide) e Formas Multibacilares - MB - (Dimorfa e Virchowiana). O esquema de tratamento padronizado Poliquimioterapia (PQT/OMS) utiliza nos pacientes paucibacilares os medicamentos dapsona e rifampicina; já para os multibacilares as drogas são dapsona, rifampicina e clofazimina ${ }^{1}$. Não foram realizados testes imunológicos.

O tratamento estatístico dos dados baseou-se em análise descritiva de dados e do teste de Wilcoxon para os dados não paramétricos. Este estudo foi aprovado pelos Comitês de Ética da Unidade Mista de Paracatu e da Universidade de Brasília (Processo de $n^{\circ} 038 / 2001$ ).

\section{Resultados}

A Figura 2 mostra que o coeficiente de detecção da hanseníase em menores de quinze anos, no município de Paracatu, apresentou, nos anos de 1994 e 1996, uma classificação de alta endemicidade e, entre os anos de 1997 e 2001, a classificação foi de município hiperendêmico.

Na Tabela 1 observa-se que a faixa etária predominante dos pacientes com diagnóstico de hanseníase ficou na idade de dez a quatorze anos (75,5\%), sendo que o grupo de faixa etária entre cinco a nove anos representava $24,5 \%$ da totalidade dos casos.

*Coeficiente de detecção anual de casos novos na população de 0 a 14 anos, por 10.000 habitantes. Hiperendêmico:>1,0/ 10.000 hab.; Muito Alto:1,0 - 0,5/10.000 hab.; Alto: 0,5 - 0,25/10.000 hab.; Médio: 0,25 - 0,05/10.000 hab.; Baixo: < 0,05/ 10.000 hab.

Annual detection coefficient of new cases in population aged 0 to 14, per 10.000 inhabitants. Hyper-endemic: > 1.0/10.000 inh.; Very High: $1.0-\mid 0.5 / 10.000$ inh.; High: $0.5-\mid 0.25 / 10.000$ inh.; Medium: $0.25-\mid 0.05 / 10.000$ inh.; Low: $<0,05 / 10,000$ inh. 


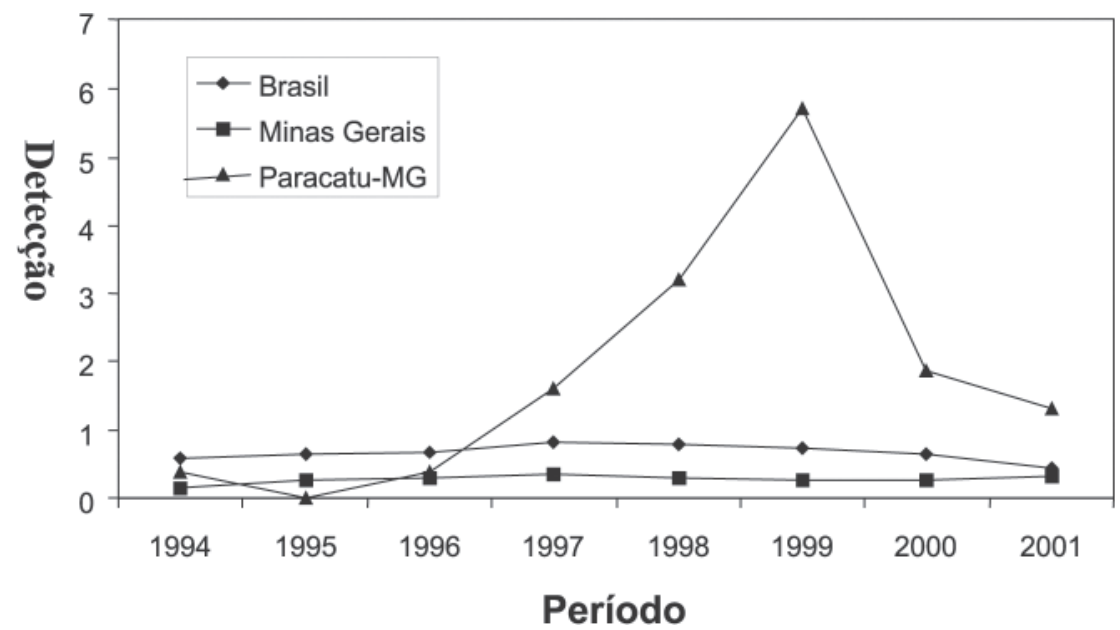

Figura 2 - Comparativo dos Coeficientes de Detecção de Hanseníase em menores de 15 anos no Brasil, em Minas Gerais e no Município de Paracatu, entre os anos de 1994 e 2001.

Figure 2 - Comparative Detection Coefficients of Leprosy in children under fifteen-years of age in Brazil, Minas Gerais and in Paracatu-MG, from 1994 to 2001.

Fonte: Ministério da Saúde, Secretaria Estadual de Saúde, Serviço de Hanseníase, 2002.

Source: Ministry of Health, Minas Gerais State Health Department, Hansen's Disease Service, 2002.

Em relação ao sexo da população deste estudo, a Tabela 1 mostra que 53,0\% dos pacientes eram do sexo masculino e $47,0 \%$ do sexo feminino.

Em relação ao esquema da vacina BCG (Tabela 1), encontrou-se entre os portadores de hanseníase estudados a presença de cicatriz vacinal em trinta e quatro pacientes (76,0\%). Somente $15,5 \%$ dos pacientes estudados apresentavam as duas cicatrizes vacinais.

O exame de baciloscopia foi realizado em $95,6 \%$ dos pacientes, com resultado positivo em $4,5 \%$ deles da forma Dimorfa.

As formas Multibacilares (56,0\%) da doença (Tabela 1) predominaram sobre as formas Paucibacilares (44,0\%).

A Tabela 1 mostra que as incapacidades* atingiram $22,0 \%$ dos doentes no diagnóstico, sendo que $20,0 \%$ dos pacientes apresentaram grau 1 e $2,0 \%$, grau 2 . Na alta, $13,0 \%$ dos pacientes mostravam incapacidades, com $2,2 \%$ dos doentes apresentando grau 1 e $11,1 \%$ registrando grau 2 . A forma clínica que mais apresentou incapacidades foi a Dimorfa, em $31,1 \%$ dos doentes com algum grau de incapacidade, seguida da forma
Tuberculóide, que acometeu 4,4\% dos doentes.

Vinte e quatro por cento dos pacientes apresentaram reações hansênicas no tratamento e $9,0 \%$ no pós-alta da forma Dimorfa.

Verificou-se que $62,0 \%$ dos pacientes hansênicos tinham contatos intradomiciliares com história de hanseníase; $20 \%$ dos doentes tinham os pais portadores da enfermidade de formas multibacilares (principalmente Virchowianos); cerca de $20,0 \%$ eram os avós e 18,0\% referiam-se à hanseníase contraída pelos tios; $71 \%$ dos contatos intradomiciliares foram examinados durante o tratamento do doente e 19,0\% após a alta. Cerca de 10,0\% dos contatos não foram avaliados conforme as recomendações do Programa de Hanseníase.

Somente em $15,5 \%$ dos contatos intradomiciliares do portador de hanseníase foram verificadas as duas cicatrizes da vacina BCG, preconizadas pelo Programa de Hanseníase.

\section{Discussão}

A população deste estudo representou $9,0 \%$ de um total de quinhentos doentes de

"Percentagem de casos com incapacidades físicas entre os casos avaliados: Alto => 10\%; Médio 5 - $10 \%$; Baixo < 5\%

Percentage of cases with physical incapacities among the cases evaluated: High $=>$ 10\%; Medium: $5-\mid 10 ;$ Low $<5 \%$ 
Tabela 1 - Distribuição da hanseníase segundo Faixa Etária, Sexo, Moradia, BCG, Classificação Operacional, Classificação Clínica, Reações e Grau de Incapacidade em portadores menores de quinze anos entre 1994 e 2001.

Table 1 - Distribution of leprosy based on Age group, Gender, Dwelling, BCG, Operational Classification, Clinical Classification, Reactions and Degree of Disability in patients under fifteen years of age, from 1994 to 2001.

\begin{tabular}{cc}
\hline FAIXA ETÁRIA & $\%$ \\
\hline 0 a 4 anos & 0,0 \\
5 a 9 anos & 24,5 \\
10 a 14 anOs & 75,5 \\
\hline SEXO & $\%$ \\
\hline MASCULINO & 53,0 \\
FEMININO & 47,0 \\
\hline MORADIA & $\%$ \\
\hline ZONA URBANA & 84,0 \\
ZONA RURAL & 16,0 \\
\hline BCG & $\%$ \\
\hline PRESENÇA DE CICATRIZ VACINAL & 76,0 \\
NÃO VERIFICADO & 24,0 \\
\hline CLASSIFICAÇÃO OPERACIONAL & $\%$ \\
\hline FORMAS PAUCIBACILARES & 44,0 \\
FORMAS MULTIBACILARES & 56,0 \\
\hline CLASSIFICAÇÃO CLINICA & $\%$ \\
\hline FORMA INDETERMINADA & 30,8 \\
FORMA TUBERCULÓIDE & 13,2 \\
FORMA DIMORFA & 56,0 \\
\hline GRAU DE INCAPACIDADE NO DIAGNÓSTICO & $\%$ \\
\hline GRAU 1 & 20,0 \\
\hline GRAU 2 & 2,0 \\
\hline GRAU DE INCAPACIDADE NA ALTA & $\%$ \\
\hline GRAU 1 & 2,2 \\
\hline GRAU 2 & 11,1 \\
\hline NO DIAGÕES & $\%, 0$ \\
\hline NO PÓS-ALTA & \\
\hline & \\
\hline
\end{tabular}

hanseníase, em todas as faixas etárias no serviço desta nosologia do Centro de Saúde Central. Este resultado está de acordo com o esperado, visto que, em 2001, as Américas tinham $8 \%$ dos casos de hanseníase em menores de quinze anos, a África tinha $11 \%$ e o Sudeste da Ásia $18 \%{ }^{24}$. O reflexo das ações de controle desenvolvidas pelo Serviço de Hanseníase de Paracatu, como treinamento de algumas equipes do Programa de Saúde da Família, e as palestras para cerca de oito mil escolares, esclarecendo sobre os sinais e sintomas da doença, provavelmente proporcionaram a descoberta dos casos existentes subnotificados.

Ao analisar o nível de escolaridade da população estudada, $95,6 \%$ freqüentavam a escola regularmente. A maioria do grupo 
estudado $(24,4 \%)$ estava cursando a terceira série do ensino fundamental, seguida de $17,8 \%$ na quinta, $15,6 \%$ na quarta e $11,0 \%$ na sexta série. Quanto ao fato de a maior parte da população deste estudo estar na faixa etária entre onze e treze anos de idade, com $49,0 \%$ dos casos, outros estudos corroboram estes achados ${ }^{7,8,}$, Por um lado, este fato sugere que a escolaridade desta população esteja defasada em relação à idade, uma vez que, nesta idade, os alunos já cursaram cerca de seis a oito anos de estudo ${ }^{25}$. Por outro lado, reflete a condição social dos sujeitos acometidos por esta enfermidade, ou seja, a situação encontrada pode refletir em exclusão social e em menor oportunidade de vida desta população em comparação com outros grupos sociais mais abastados.

Com relação ao sexo, não houve diferença estatística $(p=0,078)$ entre os acometidos masculinos e femininos cujos resultados são corroborados por outros estu$\operatorname{dos}^{10,15,20}$.

Em 24,5\% dos doentes, não foi possível verificar a presença de cicatriz vacinal, com o BCG, provavelmente devido à omissão de informações escritas pelos profissionais de saúde ou anotações em outros impressos não constantes no prontuário. O Programa Nacional de Eliminação da Hanseníase preconiza duas doses da vacina BCG para que o paciente obtenha um nível adequado de proteção contra a doença. Como somente 15,5\% dos pacientes estudados apresentavam as duas cicatrizes, concluímos que esta população apresentou baixa cobertura vacinal pelo BCG. A importância da vacinação BCG para proteção contra a hanseníase está bem documentada em diversos estudos que demonstram que a detecção da doença é menor na clientela que recebe as duas doses de $\mathrm{BCG}^{26,27}$. Durante o tratamento dos doentes, $71,0 \%$ dos seus contatos intradomiciliares foram examinados pela equipe do serviço de hanseníase. Depois do tratamento, foram examinados $19 \%$ dos comunicantes que ainda não tinham sido examinados. Do total geral, $10 \%$ dos contatos não foram examinados por se recusarem ou por terem origem desconhecida. Outro fator a ser considerado é a não descentralização do atendimento da doença para todos os serviços de saúde existentes no município, principalmente para as equipes do Programa de Saúde da Família.

Com relação ao exame de baciloscopia com resultado positivo em apenas $4,5 \%$ dos pacientes, LANA et al*, em estudo realizado em Belo Horizonte - MG, encontrou 4,8\% das lâminas com resultado positivo. No nosso estudo, muitos doentes da forma Dimorfa, que apresenta imunidade instável ${ }^{1}$, talvez poderiam ter sido classificados do pólo DT (Dimorfo-Tuberculóide). Outros pacientes também foram classificados segundo o número de lesões encontradas ${ }^{1}$. Também não se pode descartar possível subdiagnóstico de formas Indeterminada e Tuberculóide.

A forma Dimorfa (MB) acometeu 56,0\% dos doentes, seguida da Indeterminada (PB) com 30,8\% e da Tuberculóide (PB) que apresentou $13,2 \%$. Houve diferença significativa entre as formas clínicas da doença ( $\mathrm{p}=0,026)$. A maioria dos pacientes, quando diagnosticados de formas multibacilares, serve de alerta para o serviço local, pois estas são consideradas as formas contagiantes da doença $\mathrm{e}$ potencialmente incapacitantes ${ }^{1}$. Lana et al.* encontrou que $25,0 \%$ dos casos eram de formas paucibacilares e $75,0 \%$ de formas multibacilares. O estudo de Selvasekar et al. ${ }^{15}$ também encontrou que as formas multibacilares ocorreram entre crianças de maior idade, semelhante a este trabalho.

Ressalta-se, neste estudo, a doença hansênica em crianças com imunidade elevada, como $13,2 \%$ dos pacientes diagnosticados com a forma Tuberculóide da doença. Ou seja, mesmo com certa defesa imunológica específica, parte desta população adoeceu. A maioria dos pacientes sendo diagnosticados na forma Dimorfa demonstra diagnóstico tardio. Lana et al.* e Lastoria et al. ${ }^{28}$ alertam que pacientes menores de quinze anos, diagnos-

*Lana C F et al. Detecção da Hanseníase na Faixa Etária de 0 a 14 anos em Belo Horizonte no Período 92-99: Implicações para o Controle. UFMG. Belo Horizonte: [s.n]. 2002. 14p. Digitado. 
ticados já de formas polarizadas, demonstram alta prevalência da doença, pois deveriam ser diagnosticados na forma Indeterminada, que é a inicial da doença.

As reações hansênicas encontradas no diagnóstico (24,0\%) e na alta $(9,0 \%)$ mostram a necessidade de o serviço estar preparado para atender adequadamente os portadores da enfermidade em relação à prevenção de incapacidades. Hammond e Rao ${ }^{17}$, na Índia, encontraram 14,3\% de crianças com reações hansênicas e também alertaram sobre este problema.

A alta porcentagem de pacientes com incapacidades, no momento do diagnóstico, pode ser reflexo de diagnóstico tardio e também da prevalência oculta da doença ${ }^{29,30}$. Também não podem ser descartados possíveis erros de avaliação do grau de incapacidade no momento do diagnóstico ou mesmo piora do grau de incapacidade durante $o$ tratamento por questões técnicas (o setor de fisioterapia não acompanhou, mensalmente, todos os pacientes com algum grau de incapacidade no diagnóstico). Dificuldades socioeconômicas também podem ter impedido que alguns pacientes fizessem o devido acompanhamento prescrito.

Com relação ao fato de $62,0 \%$ dos pacientes terem contatos intradomiciliares com história de hanseníase, uma possível explicação desta descoberta é que a equipe do serviço de hanseníase do município é experiente, com cerca de quinze anos atendendo e conhecendo bem a comunidade local. A literatura existente apresenta vários estudos, mostrando a transmissão por contatos intra e extradomiciliares e que confirmam este achado $^{15,31}$.

Apesar da norma do Ministério da Saúde preconizar o exame de todos os contatos intradomiciliares dos doentes e da vacinação com duas doses da vacina $\mathrm{BCG}^{1}, 10,0 \%$ dos contatos não foram examinados e, em apenas $15,5 \%$ deles foram verificadas as duas cicatrizes vacinais preconizadas pelo Programa de Hanseníase, sendo a mesma pouco utilizada como prevenção da doença. As possíveis causas podem ser a recusa de alguns contatos ao exame, ou por não terem residência conhecida.

\section{Conclusões}

Os resultados apontam que o serviço de hanseníase em Paracatu realiza diagnóstico tardio devido à maioria dos pacientes serem diagnosticados de forma Multibacilar, além do elevado número de casos com algum grau de incapacidade.

Os resultados também sugerem a existência de uma prevalência oculta da doença que deve ser enfrentada, visando o diagnóstico precoce da forma inicial Indeterminada.

A vacina $B C G$, duas doses, foi pouco utilizada como medida preventiva no período estudado.

Faz-se necessário descentralizar o atendimento ao portador de hanseníase com treinamento e capacitação das equipes do Programa de Saúde da Família e profissionais de outros serviços que atuam no município, a fim de diagnosticar precocemente e tratar os casos existentes, além de implementar as atividades de prevenção e de controle da doença.

Sendo assim, os achados deste estudo mostram a necessidade de priorizar a área rural do município e demais áreas hiperendêmicas pelo fato de as mesmas não possuírem cobertura adequada dos serviços de saúde.

Por ser a hanseníase um grave problema de saúde pública no município de Paracatu - MG, outros estudos devem ser realizados a fim de ampliar o conhecimento da doença em menores de quinze anos para mudar este quadro existente na região estudada.

\section{Agradecimento}

Dra. Erika Neumann Rocha Salgueiro, FUNASA - Paracatu/MG. 


\section{Referências}

1. Brasil. Ministério da Saúde. Secretaria de Políticas de Saúde. Hanseníase: atividades de controle e manual de procedimentos. Brasília; 2001.

2. Pereira GFM. Características da hanseníase no Brasil: situação e tendência no período 1985 a 1996. [dissertação de mestrado]. São Paulo: Escola Paulista de Medicina da UNIFESP; 1999.

3. Organização Pan-Americana da Saúde (OPAS). Eliminação da Hanseníase das Américas. Boletim n. 8; 2000 .

4. Brasil. Ministério da Saúde. Programa de Eliminação da Hanseníase. Série Hístórica. Arquivos avulsos; 2004.

5. Ganapati R, Revankar CR. Associated cases in the families of scholl children with leprosy. Lepr Rev 1978; 49: 43-6.

6. Keller R, Deen RD. Leprosy in children aged 0-14 years: report of an 11- year control programme. Lepr Rev 1985; 56: 239-45.

7. Prasad PVS. Childhood Leprosy in a Rural Hospital. Indian J Pediatr 1998; 65(5): 751-4.

8. Belda W, Marlet JM. O fator idade na epidemiologia da hanseníase. Boletim de Divisão Nacional de Lepra 1973; 32(2,3,4): 60-76.

9. Lombardi C. História natural da hanseníase. In: Lombardi C. Hanseníase: epidemiologia e controle. São Paulo: Imprensa Oficial do Estado, Arquivo do Estado; 1990. p.13-20.

10. Yawalkar SJ. Leprosy for medical practitioners and paramedical workers. 1. ed. Basle: Novartis; 2002.

11. Talhari S, Neves RG. Dermatologia tropical: hanseníase. Manaus [s.n.]; 1997.

12. Duncan ME. Leprosy in young children: past, present and future. Int J Lepr Other Mycobact Dis 1985; 3: 46873.

13. El-Zawahry MP; El-Zawahry K. Child leprosy. J Egypt Med Assoc 1977; 69(5/6): 457-60.

14. Ebenezer L, Arunthati S, Kurian N. Profile of leprosy in children: past and present. Indian J Lepr 1997; 3: 2559 .

15. Selvasekar A et al. Childhood leprosy in an endemic area. Lepr Rev 1999; 1: 21-7.

16. Chen XS et al. . Leprosy in children: a retrospective study in China, 1986-1987. J Trop Pediatr 2000; 46(4): 207-11.

17. Hammond PJ, Sundar Rao PSS. The tragedy of deformity in childhood leprosy. Lepr Rev 1999; 70(2): 217-20.
18. Mahajan PM, Jogaikar DG, Mehta JM. Study of deformities in children with leprosy: an urban experience. Indian J Lepr 1995; 67(4): 405-9.

19. Bechelli LM et al. Proposed method for estimating prevalence based on rates in children. WHO Bull 1973; 48: $502-3$

20. Bryceson A, Pfaztz Graff RE. Leprosy: medicine in the tropics. London: Churchill Livingstone; 1990. p. 203-30.

21. Saha K et al. A study on nutrition, growth and development of a high-risk group of children of urban leprosy patients. Eur J Clin Nutri 1990; 44: 471-9.

22. Dayal R et al. A clinic-bacteriological profile of leprosy in children. Indian Pediatr 1995; 26(2): 122-8.

23. Opromolla DVA. História. In: Opromolla DVA. Noções de hansenologia. Bauru: Centro de Estudos "Dr. Reynaldo Quagliato”, Hospital Lauro de Souza Lima; 2000(a). p. 1-5.

24. World Health Organization. The Elimination of Leprosy as a Public Health Problem. Status Report 2001. Geneva; 2002

25. Minas Gerais. Sec. Est. Educ. CEE. Informativo mai de ensino do Estado de Minas Gerais. Parecer 1137/2000. Ed. Lancer 2001; 30-40.

26. Stanford JL et al. Vaccination and skin test studies on the children of leprosy patients. Int J Lepr Other Mycobact Dis 1989; 57(1): 38-44.

27. Zodpey S et al. Protective effect of Bacillus Calmette Guerin (BCG) against leprosy: a population-based case-control study in Nagpur, India. Lepr Rev 1999; 70(3): 287-94.

28. Lastoria JC, Macharelli CA, Putinatti MSMA. Hanseníase: realidade no seu diagnóstico clínico. Hansen Int 2003; 28(1): 53-8.

29. Ferreira J. Validade do grau de incapacidade como indicador de diagnóstico tardio da hanseníase. Hansenologia Internationalis 1998; número especial: 79-84. Trabalho apresentado no Congresso da Associação Brasileira de Hansenologia. Foz do Iguaçu; 1997.

30. Suarez REG, Lombardi C. Estimado de prevalência de lepra. Hansenologia Internationalis 1997; 22(2): 31-5.

31. Sundharam JÁ. Leprosy in childhood. Indian Pediatr 1990; 27(0): 1126-8.

recebido em: 06/07/04 versão final reapresentada em: 16/02/05 aprovado em: 25/02/05 